\title{
Adaptation to a Commercial Quaternary Ammonium Compound Sanitizer Leads to Cross-Resistance to Select Antibiotics in Listeria monocytogenes Isolated From Fresh Produce Environments
}

\author{
Rebecca Bland 1,2, Joy Waite-Cusic ${ }^{2}$, Alexandra J. Weisberg ${ }^{3}$, Elizabeth R. Riutta ${ }^{3}$, \\ Jeff H. Chang ${ }^{3}$ and Jovana Kovacevic ${ }^{1,2 *}$
}

${ }^{1}$ Food Innovation Center, Oregon State University, Portland, OR, United States, ${ }^{2}$ Department of Food Science and Technology, Oregon State University, Corvallis, OR, United States, ${ }^{3}$ Department of Botany and Plant Pathology, Oregon State University, Corvallis, OR, United States

OPEN ACCESS

Edited by:

Yasser M. Sanad

University of Arkansas at Pine Bluff,

United States

Reviewed by:

Mohit Bansal,

University of Arkansas, United States

David Nicholas,

New York State Department of Health,

United States

${ }^{*}$ Correspondence:

Jovana Kovacevic

jovana.kovacevic@oregonstate.edu

Specialty section: This article was submitted to

Food Microbiology,

a section of the journa

Frontiers in Microbiology

Received: 25 September 2021 Accepted: 06 December 2021

Published: 10 January 2022

Citation:

Bland R, Waite-Cusic J, Weisberg AJ, Riutta ER, Chang JH and Kovacevic J (2022) Adaptation

to a Commercial Quaternary

Ammonium Compound Sanitizer Leads to Cross-Resistance to Select Antibiotics in Listeria monocytogenes Isolated From Fresh Produce

Environments.

Front. Microbiol. 12:782920. doi: 10.3389/fmicb.2021.782920
The effective elimination of Listeria monocytogenes through cleaning and sanitation is of great importance to the food processing industry. Specifically in fresh produce operations, the lack of a kill step requires effective cleaning and sanitation to mitigate the risk of cross-contamination from the environment. As facilities rely on sanitizers to control L. monocytogenes, reports of the development of tolerance to sanitizers and other antimicrobials through cross-resistance is of particular concern. We investigated the potential for six L. monocytogenes isolates from fresh produce handling and processing facilities and packinghouses to develop cross-resistance between a commercial sanitizer and antibiotics. Experimental adaptation of isolates belonging to hypervirulent clonal complexes (CC2, CC4, and CC6) to a commercial quaternary ammonium compound sanitizer (CQAC) resulted in elevated minimum inhibitory concentrations (2$3 \mathrm{ppm}$ ) and minimum bactericidal concentrations (3-4 ppm). Susceptibility to cQAC was restored for all adapted (qAD) isolates in the presence of reserpine, a known efflux pump inhibitor. Reduced sensitivity to 7/17 tested antibiotics (chloramphenicol, ciprofloxacin, clindamycin, kanamycin, novobiocin, penicillin, and streptomycin) was observed in all tested isolates. GAD isolates remained susceptible to antibiotics commonly used in the treatment of listeriosis (i.e., ampicillin and gentamicin). The whole genome sequencing of $\mathrm{qAD}$ strains, followed by comparative genomic analysis, revealed several mutations in fepR, the regulator for FepA fluoroquinolone efflux pump. The results suggest that mutations in fep $R$ play a role in the reduction in antibiotic susceptibility following low level adaptation to CQAC. Further investigation into the cross-resistance mechanisms and pressures leading to the development of this phenomenon among $L$. monocytogenes isolates recovered from different sources is needed to better understand the likelihood of cross-resistance development in food chain isolates and the implications for the food industry.

Keywords: antibiotic resistance, cross-resistance, quaternary ammonium compound, sanitizers, whole genome sequencing 


\section{INTRODUCTION}

Listeria monocytogenes is a Gram-positive bacterium that remains one of the leading causes of mortality among foodborne pathogens in the United States (Scallan et al., 2011; de Noordhout et al., 2014). Listeriosis, the disease caused by $L$. monocytogenes, largely effects vulnerable populations, including pregnant women, children, elderly, and people who are immunocompromised (Hamon et al., 2006; Buchanan et al., 2017). Due to the immune status of this vulnerable population and the invasive nature of the disease, antibiotics are critical to the successful treatment of listeriosis. The first choice antibiotic for the treatment is typically ampicillin (B-lactam), alone or in combination with gentamicin (aminoglycoside) (Hof, 2004). While L. monocytogenes remains largely susceptible to a wide range of antibiotics, there have been reports of multidrug resistance in isolates recovered from food production environments (Prazak et al., 2002; Jorgensen et al., 2021).

Listeria monocytogenes is well adapted to agricultural environments, where it proliferates in decaying plant matter in the soil (Linke et al., 2014; Liao et al., 2021). It is also frequently recovered from diverse food processing environments (Gray et al., 2006; Freitag et al., 2009; Cherifi et al., 2018; Kim et al., 2018; Hurley et al., 2019; Sullivan and Wiedmann, 2020). Listeria monocytogenes prevalence in these environments presents a particular concern to processors of minimally processed ready-to-eat (RTE) products, such as fresh produce, that are prone to contamination during handling and processing (Gallagher et al., 2003; Farber et al., 2020). Often, control of L. monocytogenes in these environments relies on cleaning and sanitation programs. In the United States, sanitizers are regulated by the Environmental Protection Agency (EPA) and are required to achieve a 5-log reduction of a test organism on a food contact surface and a 3-log reduction on a non-food contact surface (Office of Chemical Safety and Pollution Prevention [OCSPP], 2012). Sanitizers are formulated at concentrations that will deliver a bactericidal effect, and in general many-fold higher than the minimum bactericidal concentration (MBC) for foodborne pathogens of concern (Cruz and Fletcher, 2012).

Quaternary ammonium compounds (QACs) are one group of sanitizers commonly used in the food industry. QACs are cationic antimicrobials with medium-to-long alkyl side chains (Gilbert and Moore, 2005; Wessels and Ingmer, 2013). The mechanism of bacterial cell inhibition is generally thought to be by the hydrophobic chain interpolating into the lipid bilayer of the cellular membrane leading to issues in osmoregularity and leaking of cell contents. The manufacturer recommended concentrations (MRCs) for commercial QAC (cQAC) application range from 200 to $800 \mathrm{ppm}$ (Cruz and Fletcher, 2012; Boucher et al., 2021). The reported minimum inhibitory concentrations (MICs) for L. monocytogenes to QACs range from $\leq 2$ ppm (Romanova et al., 2006; Martínez-Suárez et al., 2016; Møretrø et al., 2017; Yu et al., 2018; Roedel et al., 2019) to 40 ppm (Elhanafi et al., 2010; Dutta et al., 2013). Ideally, appropriate cleaning and sanitation procedures are paired with optimum hygienic design of the facility and equipment; however, this ideal combination is rare, particularly in fresh
TABLE 1 | Genetic profiles and characterization of Listeria monocytogenes isolates $(n=6)$ selected for evaluation of cross-resistance.

\begin{tabular}{lccccc}
\hline Isolate no. & $\begin{array}{c}\text { Sequence } \\
\text { type }\end{array}$ & $\begin{array}{c}\text { Clonal } \\
\text { complex }\end{array}$ & LIPI-3 & LIPI-4 & inlA $^{\mathbf{a}}$ \\
\hline WRLP354 & 2 & 2 & - & - & + \\
WRLP380 & 2 & 2 & - & - & + \\
WRLP394 & 219 & 4 & + & + & + \\
WRLP483 & 219 & 4 & + & + & + \\
WRLP530 & 6 & 6 & + & - & $3-\operatorname{cod} \Delta$ \\
WRLP533 & 6 & 6 & + & - & $3-\operatorname{cod} \Delta$ \\
\hline
\end{tabular}

All strains were isolated from produce operations in the Pacific Northwest during 2018-2019 by Jorgensen et al. (2020).

ainlA 3-cods indicates a 3-codon deletion in amino acid positions 738-740 (aspartic acid, threonine, and serine).

produce facilities. These less-than-ideal environments can create scenarios where L. monocytogenes could be exposed to sublethal sanitizer concentrations. Links between reduced susceptibility to QACs and antibiotic resistance have been reported for various foodborne bacteria, including L. monocytogenes (Heir et al., 1999; Braoudaki and Hilton, 2004; Langsrud et al., 2004; Rakic-Martinez et al., 2011; Gnanadhas et al., 2013; Kovacevic et al., 2013; Bansal et al., 2018; Kode et al., 2021). This phenomenon of cross-resistance can occur when microorganisms develop survival methods that are effective against different antimicrobial agents with similar mechanisms of action (SCENIHR, 2009). For example, a mutation or upregulation of a gene initiated by adaptation or exposure to one of the antimicrobials may subsequently affect the efficacy of another antimicrobial agent (Jiang et al., 2018; Amsalu et al., 2020).

We previously evaluated the prevalence and distribution of antimicrobial resistance (AMR) in L. monocytogenes from produce handling and processing facilities in the Pacific Northwest (Jorgensen et al., 2021). These isolates have diverse antibiogram profiles and represent clonal complexes associated with hypervirulent phenotypes. The present study investigated the potential for antibiotic crossresistance to develop in these strains following adaptation to a commercial sanitizer (cQAC). Culture-based assays and whole genome sequence (WGS) comparisons were used to evaluate differences between the wild-type (WT) and cQAC-adapted (qAD) strains.

\section{MATERIALS AND METHODS}

\section{Bacterial Strains and Sanitizer}

A total of six L. monocytogenes isolates previously recovered from produce packing, processing, and handling environments in the Pacific Northwest (Jorgensen et al., 2020, 2021) were selected for adaptation to CQAC (Table 1). All isolates were previously serogrouped, multi-locus sequence typed and assessed for AMR using a standard disk diffusion assay (CLSI, 2015; Jorgensen et al., 2021). Isolates used in this study were selected based on their AMR profiles and multi-locus sequence types 
(MLST). Specifically, up to two isolates belonging to each available hypervirulent clonal complex (CC) 2, CC4, and CC6 (Maury et al., 2016), and with unique AMR profiles (when available) were included in this study. Isolates were stored at $-80^{\circ} \mathrm{C}$ in trypticase soy broth (TSB; Acumedia, Neogen, Lansing, MI, United States) with 25\% (v/v) glycerol. Prior to use, isolates were resuscitated on trypticase soy agar (TSA; Acumedia) with incubation at $35^{\circ} \mathrm{C}$ for $24 \mathrm{~h}$ and used for a maximum of 2 weeks.

The sanitizer used in this study was a commercial quaternary ammonium compound, cQAC (1-6 ppm; Professional Lysol No Rinse Sanitizer; EPA registration 675-30; Reckitt Benckiser, Parsippany, NJ, United States). Stock solution of the cQAC sanitizer was prepared in accordance with the manufacturer recommended concentration (MRC; $200 \mathrm{ppm}$ ), filter sterilized, and stored for up to 1 week at $4^{\circ} \mathrm{C}$.

\section{Minimum Inhibitory and Minimum Bactericidal Concentration}

Microbroth dilution assay described by Boucher et al. (2021) was used to assess minimum inhibitory (MICs) and minimum bactericidal concentration (MBCs) of cQAC, with minor modifications. Briefly, a single colony was transferred to TSB $(5 \mathrm{ml})$ and incubated at $30^{\circ} \mathrm{C}$ for $16 \mathrm{~h}$, with shaking $(150 \mathrm{rpm}$; Thermo Scientific, MaxQ4000, Waltham, MA, United States). Following incubation, each culture was diluted to approximately $7 \log \mathrm{CFU} / \mathrm{ml}$ in $0.1 \%$ peptone water (Fisher; Hampton, NH, United States). Inoculum was confirmed on TSA incubated at $35^{\circ} \mathrm{C}$ for $24 \mathrm{~h}$ using the track dilution method by Jett et al. (1997). Diluted cultures were added to TSB with $0.6 \%$ yeast extract (TSB-YE; Acumedia) containing 1, 2, 3, 4, 5, or 6 ppm CQAC (i.e., diluted from MRC stock solution) at approximately $5 \mathrm{log}$ CFU/ml in a final volume of $10 \mathrm{ml}$. An aliquot $(200 \mu \mathrm{l})$ of each culture/sanitizer mixture was transferred to a sterile 96well plate (VWR; Radnor, PA, United States), in duplicate. Plates were incubated at $30^{\circ} \mathrm{C}$ in a SpectraMax plate reader (Molecular Devices). $\mathrm{OD}_{600}$ was measured at $30 \mathrm{~min}$ interval for $24 \mathrm{~h}$ with $5 \mathrm{~s}$ of shaking prior to measurement. The $\mathrm{OD}_{600}$ data were fitted to growth curves to obtain the lag-phase duration (LPD), maximum growth rate (MGR), and maximum density, using the DMFit 3.0 Excel add-in program (ComBase; Computational Microbiology Research Group, Institute of Food Research, Colney, Norwich, United Kingdom), based on the models of Baranyi and Roberts (1994). Growth curve experiments were performed at least three times. For each isolate a cut off value of 0.1 maximum $\mathrm{OD}_{600}$ was used to define inhibition. The lowest concentration with a $\max$ $\mathrm{OD}_{600}$ of $<0.1$ was interpreted as the MIC. In addition, MICs of WT and $\mathrm{qAD}$ strains were tested in the presence of reserpine (Alfa Aesar, Tewksbury, MA, United States), a known efflux inhibitor (Godreuil et al., 2003; Kovacevic et al., 2016). A working solution of reserpine $(1,000 \mu \mathrm{g} / \mathrm{ml})$ was prepared in dimethyl sulfoxide (DMSO; VWR) prior to MIC assay and stored at $4^{\circ} \mathrm{C}$ for $24 \mathrm{~h}$. Uninoculated sanitizer and sterile media controls were included in each replicate and their $\mathrm{OD}_{600}$ values served as the baseline for the sanitizer treatment. Following $24 \mathrm{~h}$ incubation in the plate reader, each well was streaked onto TSA with $0.6 \%$ yeast extract (TSA-YE; Acumedia) and incubated at $35^{\circ} \mathrm{C}$ for $24 \mathrm{~h}$. The lowest CQAC concentration where no growth was observed was considered the MBC.

Minimum inhibitory concentration of all WT and qAD isolates for ciprofloxacin was also measured using the VITEK system (Biomerieux, France; card: AST-GP75) according to manufacturer instructions.

\section{Experimental Adaptations to Sublethal Concentrations of Sanitizers}

Listeria monocytogenes strains were experimentally adapted to increasing concentrations of cQAC sanitizer. Strains were subcultured at $30^{\circ} \mathrm{C}$ with progressively higher concentrations of cQAC, alternating between TSB-YE ( $2 \mathrm{ml}, 150 \mathrm{rpm}$ shaking), and TSA-YE plates (Figure 1). Adaptations started in TSB-YE at a concentration of $1 \mathrm{ppm}$. Once the culture was visibly turbid, $10 \mu \mathrm{l}$ was transferred into fresh TSB-YE and TSA-YE media with cQAC (2 ml total volume for TSB-YE and $15 \mathrm{ml}$ for TSA-YE plates). Concentration of CQAC present in the media was increased by 1 ppm following stabilization of each incremental adaptation in TSB-YE. Once growth was observed on TSA-YE, a single colony was transferred to TSB-YE at the same concentration to stabilize the adaptation at each increment. Adaptations were stopped when no growth was visually observed after 5 days of incubation at $30^{\circ} \mathrm{C}$ in TSB-YE. The adaptations scheme used in the isolates reported here is depicted in Figure 1. For each cQAC concentration adaptation (1,2, and $3 \mathrm{ppm})$, strains were stabilized by five passages in TSB-YE with appropriate cQAC concentration. Adapted strains were frozen in TSB supplemented with CQAC at half the adapted concentration with 25\% (v/v) glycerol. TSA-YE supplemented with 3 ppm cQAC was used to revive CQAC-adapted cultures from frozen stock for use in the following assays.

\section{Whole Genome Sequencing of Commercial Quaternary Ammonium Compound Adapted Isolates}

Commercial quaternary ammonium compound adapted L. monocytogenes isolates ( $\mathrm{qAD}$ ) were revived from frozen stock on TSA-YE supplemented with 3 ppm cQAC and incubated at $35^{\circ} \mathrm{C}$ for $24 \mathrm{~h}$. A single colony was transferred to $3 \mathrm{ml}$ TSB and incubated at $35^{\circ} \mathrm{C}$ for $18-20 \mathrm{~h}$ with shaking (150 rpm). DNA was extracted using Qiagen Blood and Tissue kit (Qiagen, Germantown, MD, United States) according to the manufacturer recommendations for Gram-positive bacteria. Whole genome sequencing (WGS) libraries were prepared with the Nextera XT DNA sample preparation kit (Illumina, San Diego, CA, United States), according to the manufacturer's protocol. Pairedend sequencing $(2 \times 150 \mathrm{bp})$ was performed on the Illumina MiSeq by the Center for Quantitative Life Sciences (CQLS, Oregon State University, Corvallis, OR, United States). Raw sequence reads were quality checked with FastQC, followed by quality trimming with Trimmomatic (v 0.39). Reads were de novo assembled using SPAdes optimized with unicycler (v 0.4.8) and assemblies were annotated with Prokka (v 1.14.6). Mutations were identified by mapping the reads of the adapted 


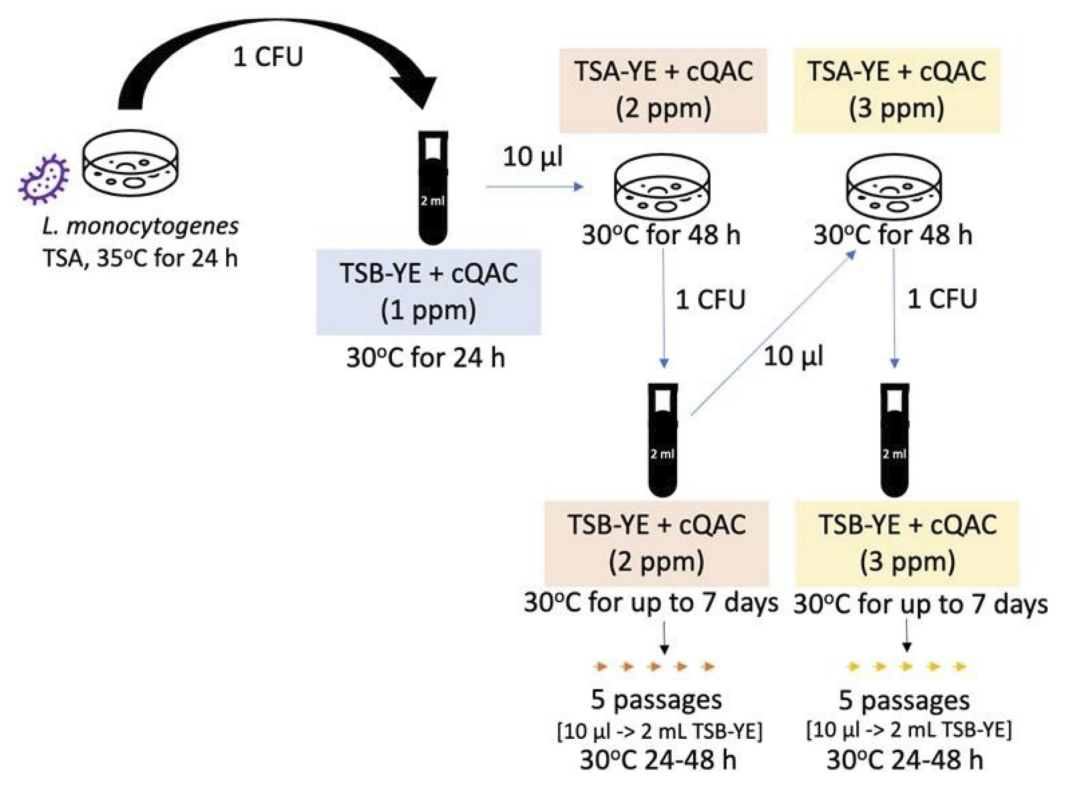

FIGURE 1 | Experimental scheme used to adapt $L$. monocytogenes to CQAC.

isolates to draft assemblies of the respective wild types. Briefly, raw reads were mapped to the respective reference sequence using BWA ( $\mathrm{v}$ 0.7.17). Alignments were annotated, sorted and duplicate reads identified with Picard tools (v 2.0.1). Graphtyper (v 2.6.2) was run on each dataset with the default parameters (Eggertsson et al., 2017). Single nucleotide polymorphisms (SNP) were filtered using vcffilter in vcflib (v 1.0.0). SNP calls annotated as "FAIL" or "heterozygous" were filtered to "nocall". SnpEff (v 4.3t) with the parameters "-no-downstream -no-upstream -no-intron" was used to predict functional effects of each SNP (Cingolani et al., 2012). Geneious (v 2020.1.2) was used to further investigate mutations and genes with identified mutations were aligned using MUSCLE (Edgar, 2004). Sequences for adapted and WT isolates are available under SRA BioProject PRJNA771688.

\section{Antibiotic Susceptibility Disk Diffusion Assay}

Following sanitizer adaptations, disk diffusion assays were used to determine if sanitizer adaptations affected sensitivity to a panel of 17 antibiotics (BBL Sensi-Disc, BD Diagnostics, Sparks, MD, United States). The tested antibiotic disks included: amikacin (AMK; $30 \mu \mathrm{g}$ ), ampicillin (AMP; $10 \mu \mathrm{g}$ ), cefoxitin (FOX; $30 \mu \mathrm{g}$ ), chloramphenicol (CHL; $30 \mu \mathrm{g}$ ), ciprofloxacin (CIP; $5 \mu \mathrm{g}$ ), clindamycin (CLI; $2 \mu \mathrm{g}$ ), erythromycin (ERY; $15 \mu \mathrm{g}$ ), gentamicin (GEN; $10 \mu \mathrm{g}$ ), kanamycin (KAN; $30 \mu \mathrm{g}$ ), novobiocin (NOV; $30 \mu \mathrm{g}$ ), penicillin $\mathrm{G}$ (PEN; $10 \mu \mathrm{g}$ ) rifampicin (RIF; $5 \mu \mathrm{g}$ ), streptomycin (STR; $10 \mu \mathrm{g}$ ), cotrimoxazole (SXT; 1.25/23.75 $\mu \mathrm{g}$ ), imipenem (IMP; $10 \mu \mathrm{g}$ ) tetracycline (TET; $30 \mu \mathrm{g}$ ), and vancomycin (VAN; $5 \mu \mathrm{g}$ ). Disk diffusion assays were carried out as described by Jorgensen et al. (2021). The diameter of each zone of inhibition was measured to the nearest $\mathrm{mm}$. Interpretation of antibiotic susceptibility (sensitive, intermediate, and resistant)
TABLE 2 | Minimum inhibitory concentrations (MIC) and minimum bactericidal concentrations (MBC) of a commercial quaternary ammonium compound sanitizer (cQAC) for wild-type (WT) and cQAC-adapted (qAD; 3 ppm) L. monocytogenes strains in the absence or presence of reserpine $(+\mathrm{R})$.

\begin{tabular}{|c|c|c|c|c|}
\hline \multirow[t]{2}{*}{ Isolate no. } & \multicolumn{4}{|c|}{ Concentration (ppm) ${ }^{a}$} \\
\hline & $\mathbf{W} T^{b}$ & $\mathbf{W T}+\mathbf{R}^{\mathrm{c}}$ & qAD & $q A D+R$ \\
\hline \multicolumn{5}{|l|}{ WRLP354 } \\
\hline $\mathrm{MIC}^{\mathrm{b}}$ & 2 & $<1$ & 3 & 2 \\
\hline $\mathrm{MBC}^{\mathrm{b}}$ & 3 & 3 & 4 & 3 \\
\hline \multicolumn{5}{|l|}{ WRLP380 } \\
\hline MIC & 2 & $<1$ & 3 & 2 \\
\hline $\mathrm{MBC}$ & 3 & 3 & 4 & 3 \\
\hline \multicolumn{5}{|l|}{ WRLP394 } \\
\hline MIC & 2 & $<1$ & 3 & 2 \\
\hline $\mathrm{MBC}$ & 3 & 3 & 4 & 3 \\
\hline \multicolumn{5}{|l|}{ WRLP483 } \\
\hline MIC & 2 & $<1$ & 3 & 2 \\
\hline $\mathrm{MBC}$ & 3 & 3 & 4 & 3 \\
\hline \multicolumn{5}{|l|}{ WRLP530 } \\
\hline MIC & 2 & $<1$ & 3 & 2 \\
\hline $\mathrm{MBC}$ & 3 & 3 & 4 & 3 \\
\hline \multicolumn{5}{|l|}{ WRLP533 } \\
\hline MIC & 2 & $<1$ & 3 & 2 \\
\hline $\mathrm{MBC}$ & 3 & 3 & 4 & 3 \\
\hline
\end{tabular}

a Manufacturer recommended concentration (MRC) for the cQAC is $200 \mathrm{ppm}$.

${ }^{b}$ Reported MIC and MBC tested in stepwise increments over three biological replicates.

In the presence of reserpine, WT isolates did not grow at the lowest dose of 1 ppm $C Q A C$.

was determined in accordance with the Clinical Laboratory Standards Institute criteria (CLSI, 2015) and compared to the measurements and susceptibility classifications established by 
TABLE 3 | Average lag phase duration, maximum growth rate, and maximum optical density of wild type (WT) and cQAC-adapted (qAD) L. monocytogenes strains exposed to sublethal concentration of CQAC ( $2 \mathrm{ppm}$ ) in tryptic soy broth with yeast extract (TSB-YE), with and without reserpine (R; $20 \mu \mathrm{g} / \mathrm{ml})$, at $30^{\circ} \mathrm{C}$ for $24 \mathrm{~h}$.

\begin{tabular}{|c|c|c|c|c|c|c|}
\hline \multirow[t]{2}{*}{ Isolate and treatment } & \multicolumn{2}{|c|}{ Lag-phase duration (h) ${ }^{a, b}$} & \multicolumn{2}{|c|}{ Maximum growth rate (increase in $\left.\mathrm{OD}_{600} / \mathrm{h}\right)^{a, c}$} & \multicolumn{2}{|c|}{ Maximum $\mathrm{OD}_{600}{ }^{a}$} \\
\hline & $\mathrm{WT}^{d}$ & qAD & WT & qAD & WT & qAD \\
\hline \multicolumn{7}{|l|}{ WRLP354 } \\
\hline TSB-YE & $8.43 \pm 0.36$ & $8.95 \pm 0.19$ & $0.17 \pm 0.00$ & $0.13 \pm 0.01^{\star a}$ & $0.59 \pm 0.02$ & $0.56 \pm 0.03$ \\
\hline $\mathrm{TSB}-\mathrm{YE}+\mathrm{R}^{f}$ & $10.49 \pm 0.06$ & $10.53 \pm 0.05$ & $0.12 \pm 0.01^{\# c}$ & $0.12 \pm 0.01$ & $0.49 \pm 0.07$ & $0.49 \pm 0.06$ \\
\hline 2 ppm cQAC & $-g$ & $10.50 \pm 1.30$ & - & $0.14 \pm 0.01$ & - & $0.50 \pm 0.01$ \\
\hline 2 ppm cQAC + R & - & - & - & - & - & - \\
\hline \multicolumn{7}{|l|}{ WRLP380 } \\
\hline TSB-YE & $8.34 \pm 0.38$ & $8.37 \pm 0.43$ & $0.16 \pm 0.01$ & $0.12 \pm 0.02$ & $0.60 \pm 0.03$ & $0.60 \pm 0.03$ \\
\hline TSB-YE + R & $9.96 \pm 0.46$ & $10.32 \pm 0.38$ & $0.13 \pm 0.01$ & $0.13 \pm 0.01$ & $0.52 \pm 0.03$ & $0.52 \pm 0.03$ \\
\hline 2 ppm cQAC & - & $10.48 \pm 1.86$ & - & $0.13 \pm 0.02$ & - & $0.55 \pm 0.0$ \\
\hline $2 p p m$ cQAC + R & - & - & - & - & - & - \\
\hline \multicolumn{7}{|l|}{ WRLP394 } \\
\hline TSB-YE & $8.08 \pm 0.40$ & $8.01 \pm 1.09$ & $0.17 \pm 0.01$ & $0.11 \pm 0.03$ & $0.65 \pm 0.02$ & $0.67 \pm 0.02$ \\
\hline TSB-YE + R & $10.40 \pm 0.19$ & $10.46 \pm 0.16$ & $0.14 \pm 0.01^{\#}$ & $0.14 \pm 0.01$ & $0.54 \pm 0.04$ & $0.54 \pm 0.03$ \\
\hline 2 ppm cQAC & - & $9.95 \pm 2.48$ & - & $0.13 \pm 0.04$ & - & $0.59 \pm 0.03$ \\
\hline 2 ppm cQAC + R & - & - & - & - & - & - \\
\hline \multicolumn{7}{|l|}{ WRLP483 } \\
\hline TSB-YE & $8.24 \pm 0.40$ & $8.41 \pm 0.34$ & $0.16 \pm 0.01$ & $0.15 \pm 0.02$ & $0.59 \pm 0.00$ & $0.56 \pm 0.03$ \\
\hline TSB-YE + R & $9.85 \pm 0.23$ & $9.58 \pm 0.19$ & $0.13 \pm 0.02$ & $0.13 \pm 0.02$ & $0.48 \pm 0.02$ & $0.48 \pm 0.01$ \\
\hline 2 ppm cQAC & - & $10.41 \pm 0.25^{\$ b}$ & - & $0.15 \pm 0.02$ & - & $0.50 \pm 0.02$ \\
\hline 2 ppm cQAC + R & - & - & - & - & - & - \\
\hline \multicolumn{7}{|l|}{ WRLP530 } \\
\hline TSB-YE & 8.58 ( 0.25 & $9.04(0.16$ & $0.16(0.01$ & $0.14(0.01$ & 0.64 ( 0.03 & 0.59 ( 0.04 \\
\hline TSB-YE + R & $10.29 \pm 0.37$ & $10.07 \pm 0.30$ & $0.13 \pm 0.02$ & $0.13 \pm 0.01$ & $0.52(0.01$ & 0.52 ( 0.01 \\
\hline 2 ppm cQAC & - & $11.66 \pm 0.11^{\$}$ & - & $0.14 \pm 0.02$ & - & $0.53 \pm 0.00$ \\
\hline 2 ppm cQAC + R & - & - & - & - & - & - \\
\hline \multicolumn{7}{|l|}{ WRLP533 } \\
\hline TSB-YE & $8.41(0.41$ & 8.99 ( 0.12 & 0.16 ( 0.01 & $0.14(0.01$ & 0.65 ( 0.02 & 0.61 ( 0.06 \\
\hline TSB-YE + R & $10.62 \pm 0.23$ & $10.73 \pm 0.19$ & $0.13 \pm 0.02$ & $0.13 \pm 0.01$ & $0.51 \pm 0.02$ & $0.51 \pm 0.01$ \\
\hline 2 ppm cQAC & - & $10.98 \pm 0.32^{\$}$ & - & $0.12 \pm 0.05$ & - & $0.54 \pm 0.03$ \\
\hline $2 \mathrm{ppm} c Q A C+R$ & - & - & - & - & - & - \\
\hline
\end{tabular}

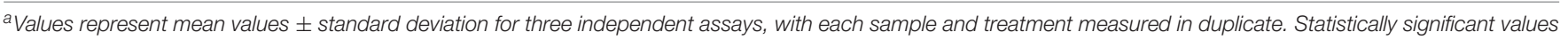
between WT and QAD strains within each treatment are indicated by asterisk ${ }^{*} P<0.05$, unpaired two-tailed $t$ test).

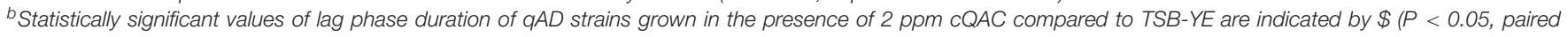
two-tailed t test).

cStatistically significant values of maximum growth rate of WT strains grown in TSB-YE compared to TSB-YE $+R$ are indicated by \# (P $<0.05$, paired two-tailed t test).

'WT, wild type.

${ }^{e} q A D$, strains adapted to 3 ppm cQAC, a commercial quaternary ammonium compound sanitizer.

${ }^{f} R$, reserpine added at $20 \mu \mathrm{g} / \mathrm{ml}$ concentration.

"No growth is indicated by "-".

The bold values represent statistically significant results.

Jorgensen et al. (2021) for each tested isolate (Supplementary Table 1). Listeria monocytogenes isolates displaying resistance to specific antibiotics were confirmed with up to two disk diffusion assays. In all assays Escherichia coli ATCC 35218 and Staphylococcus aureus ATCC 25923 were used as control strains. All results were compared with previously determined zones of inhibition for the WT strains described by Jorgensen et al. (2021).

\section{Statistical Analysis}

Comparisons between WT and qAD isolates within a treatment was performed using an unpaired, two-tail $t$ test, while a comparison between a treatment (e.g., TSB-YE $+\mathrm{R}$, or $2 \mathrm{ppm}$ $\mathrm{CQAC}$ ) and control (TSB-YE) for WT or $\mathrm{qAD}$ isolates was performed using a paired two-tailed $t$ test in Excel. For all analyses, differences were considered significant if the $P$ value was $<0.05$.

\section{RESULTS}

\section{Commercial Quaternary Ammonium Compound Adaptations for Listeria monocytogenes}

All six L. monocytogenes isolates were successfully adapted to tolerate an additional $1 \mathrm{ppm}$ of $\mathrm{CQAC}$ as determined by MIC (increased from 2 to $3 \mathrm{ppm}$ ) and $\mathrm{MBC}$ (increased from 3 to 


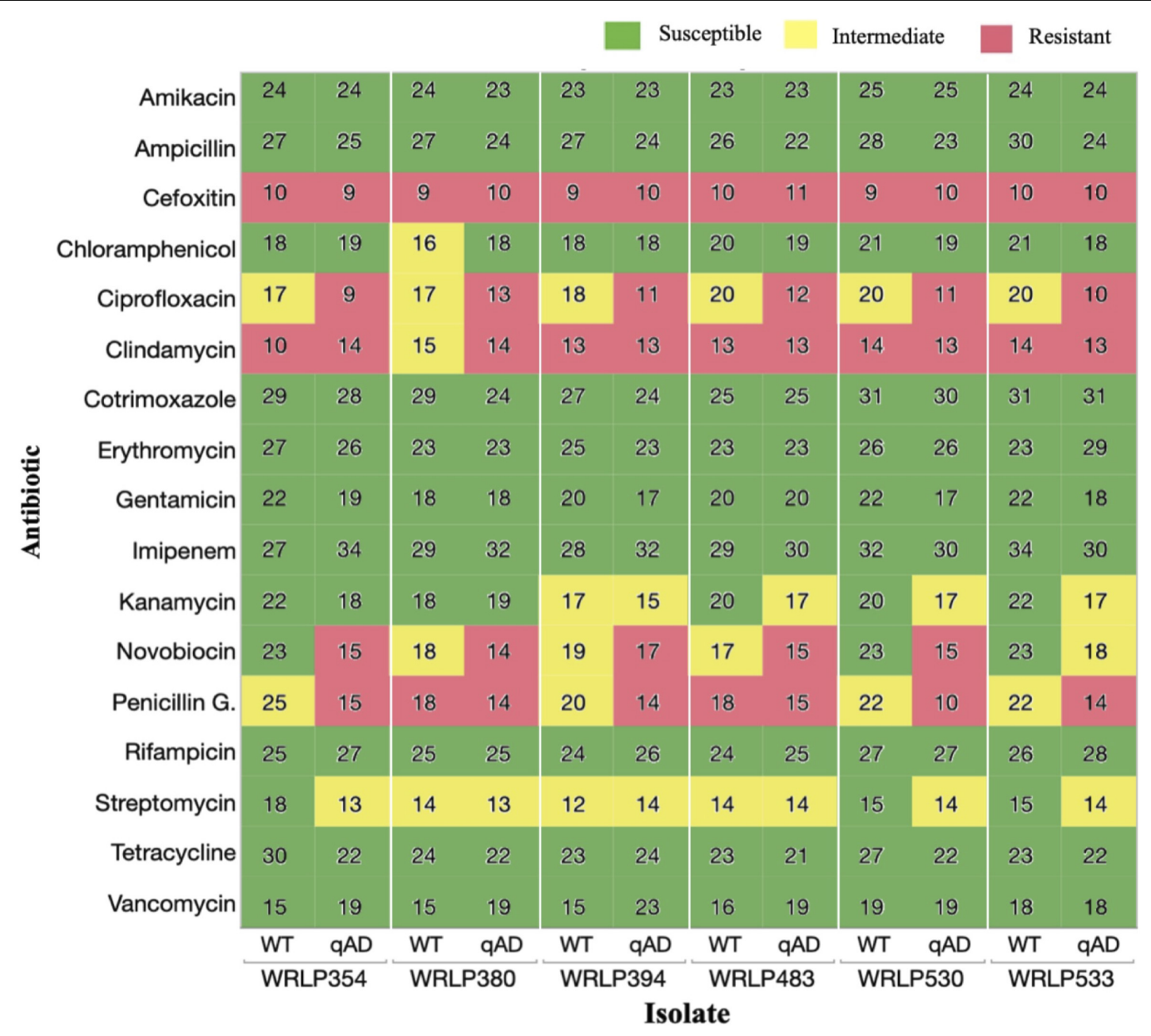

FIGURE 2 | Antibiotic susceptibility of wild-type (WT) and cQAC adapted (qAD) Listeria monocytogenes strains ( $n=6)$ to 17 antibiotics. Values reported represent zone diameters measured in $\mathrm{mm}$. For adapted isolates, the median of 2-3 independent replicates is reported. Susceptibility (green), intermediate resistance (yellow), and resistance (red) classifications, as determined by the CLSI standards for L. monocytogenes and previously reported literature, are illustrated by different colors.

4 ppm) assays (Table 2). Further attempts to adapt to higher concentrations of cQAC were not successful. In the presence of reserpine, a known efflux pump inhibitor, the $\mathrm{MIC}$ and $\mathrm{MBC}$ of all qAD strains were identical to the non-adapted WT strains. Reserpine had no effect on the MBC of WT strains; however, the WT strains were no longer able to grow in the presence of $1 \mathrm{ppm}$ of cQAC (lowest concentration tested).

Growth properties of WT and qAD L. monocytogenes strains in TSB-YE with or without CQAC (2 ppm) and/or reserpine $(20 \mu \mathrm{g} / \mathrm{ml})$ are described in Table 3. All WT and qAD strains were comparable in lag phase duration (LPD; h) and maximum cell density $\left(\mathrm{OD}_{600}\right)$ when grown in standard TSB-YE $(P>0.05)$. One qAD strain (WRLP354) had a significantly slower $(P<0.05)$ maximum growth rate in TSB-YE $\left(0.13 \pm 0.01 \mathrm{OD}_{600} / \mathrm{h}\right)$ when compared to its respective WT strain $\left(0.17 \mathrm{OD}_{600} / \mathrm{h}\right)$ (Table 3, indicated by $\left.{ }^{*}\right)$. The addition of reserpine in TSB-YE led to a slight increase in the LPD for both WT (1.62-2.32 h) and qAD (1.17-2.45 h) strains, though this was not statistically significant. Reserpine did not seem to impact the growth rate of the majority of WT and qAD isolates. The only exception were WT WRLP354 and WRLP394 strains, which grew slower in the presence of reserpine compared to their growth in TSB-YE $(P<0.05$; Table 3 , indicated by \#). There was no statistical difference between maximum $\mathrm{OD}_{600 \mathrm{~nm}}$ of WT and qAD strains (Table 3).

The addition of 2 ppm cQAC (equivalent to MIC) to TSBYE inhibited the growth of all WT L. monocytogenes strains. All qAD strains grew under these conditions; however, the LPD was significantly longer $(1.55-2.62 \mathrm{~h} ; P<0.05)$ in $3 / 6$ isolates (WRLP483, WRLP530, and WRLP533; Table 3, indicated by $\$$ ) compared to their growth in TSB-YE (i.e., without cQAC). However, there were no significant differences in maximum $\mathrm{OD}_{600} \mathrm{~nm}$ for any of the tested isolates when grown in the presence of 2 ppm cQAC compared to TSB-YE. None of the qAD strains were able to grow in the presence of $2 \mathrm{ppm}$ CQAC and reserpine. Two of the qAD strains, both representing CC2 (WRLP354 and WRLP380), were capable of growth in the presence of 3 ppm cQAC, with LPD > $19.5 \mathrm{~h}$ (data not shown). However, when both strains were exposed to 3 ppm cQAC with reserpine, their growth was inhibited. 
TABLE 4 | Minimum inhibitory concentrations (MIC) of ciprofloxacin for wild-type (WT) and cQAC-adapted (qAD; 3 ppm cQAC) L. monocytogenes strains.

\begin{tabular}{lcc}
\hline Isolate no. & \multicolumn{2}{c}{ Concentration CIP $(\mu \mathrm{l} / \mathbf{m l})$} \\
\cline { 2 - 3 } & $\mathbf{W T}$ & qAD \\
\hline WRLP354 & 1 & $>8$ \\
WRLP380 & 0.5 & 2 \\
WRLP394 & 1 & $>8$ \\
WRLP483 & 1 & $>8$ \\
WRLP530 & 1 & 4 \\
WRLP533 & 1 & 4 \\
\hline
\end{tabular}

\section{Antibiotic Susceptibility of Commercial Quaternary Ammonium Compound-Adapted Strains}

Isolates adapted to cQAC (qAD) over five serial passages resulted in changes in antibiotic susceptibility to 7/17 antibiotics tested, including CHL, CIP, CLI, KAN, NOV, PEN, and STR (Figure 2). All six $\mathrm{qAD}$ isolates resulted in varying degrees of antibiogram profile changes, as seen in the color changes between WT and qAD isolates in Figure 2. However, no obvious difference in cross-resistance patterns was seen among the three CCs evaluated.

Following adaptation to CQAC, no isolate appeared to have more advantage in the development of cross-resistance toward tested antibiotics. Isolates WRLP530 and WRLP533 exhibited changes in susceptibility to 5/17 antibiotics; followed by WRLP354, which exhibited changes in susceptibility to $4 / 17$ antibiotics; while shifts in AMR profiles for 3/17 antibiotics were seen in isolates WRLP380, WRLP394, and WRLP483 (Figure 2).

All WT isolates possessing intermediate resistance to PEN (4/17) resulted in reduced susceptibility and shift in resistance classification, based on the zone of inhibition, following adaptation to cQAC (Figure 2). With the exception of one isolate (WRLP380), all WT strains possessed resistance to CLI. Following adaptation to CQAC, WRLP380 profile changed from intermediate to resistant to CLI. Similarly, all WT isolates (6/6) that initially had intermediate resistance to CIP, became resistant following CQAC adaptation (Figure 2). Due to the consistency in profile changes across the isolates for CIP, the MIC was measured to confirm the changes observed with the disk diffusion. Isolate pairs (WT and $\mathrm{qAD}$ ) had MIC differences ranging from 0.5 to $2 \mu \mathrm{l} / \mathrm{ml}$ and 1 to $>8 \mu \mathrm{l} / \mathrm{ml}$ (Table 4 ).

In contrast, varying degrees of susceptibility shifts were seen amongst isolates for NOV before and after cQAC adaptations. Of the antibiotics where cross-resistance was observed, NOV was the only one to have WT classifications in the susceptible, intermediate, and resistant category. Following adaptation, isolates initially susceptible (WRLP354 and WRLP530) and intermediate (WRLP380 and WRLP394, WRLP483) to NOV became resistant. None of the isolates evaluated possessed resistance to STR or CHL prior to or following cQAC adaptation. While there was a reduction in susceptibility for STR in 3/6 isolates following adaptation, only one adapted isolate resulted in a susceptibility change in CHL (WRLP380; Figure 2). In contrast to other susceptibility changes, this shift resulted in increased susceptibility to CHL for WRLP380.

\section{Genomic Analysis to Identify Differences in Wild-Type and Commercial Quaternary Ammonium Compound-Adapted Listeria monocytogenes}

Comparisons of whole genome sequences of each qAD and WT pair revealed between 0 and 4 confidently called deletions and SNPs predicted to reflect mutations. Some of these SNPs were found to be in non-coding regions within the genome (Table 5). Notably, SNPs in the fepR gene were seen in $5 / 6$ isolates. This gene is predicted to encode a transcriptional regulator of FepA, a multidrug efflux pump. In three isolates with $f e p R$ mutations (WRLP380, WRLP483, and WRLP533), a SNP is predicted to cause a change in an amino acid. In WRLP394 and WRLP483, the SNP is predicted to result in a premature stop codon (PMSC) at position 141 and 175, respectively, truncating the protein by 55 and 21 amino acids, respectively. Deletions ranging from 1 to 33 bp were identified in the other three isolates (WRLP394, WRLP530, and WRLP533). The largest deletion (33 bp) is in WRLP530; the only genetic alteration observed in this isolate. Notably, no two isolates had a deletion at the exact same location within the fepR gene (Table 5).

\section{DISCUSSION}

Produce processing facilities, which have frequent turnover of raw agricultural products, present a unique risk for L. monocytogenes contamination. In these environments, L. monocytogenes is largely controlled through effective cleaning and sanitation practices. Previous reports of sanitizer and antibiotic cross-resistance have proposed broader consequences for lapses in sanitation efficacy in the food industry. In the present study, we demonstrated the ability of L. monocytogenes strains, including those representing hypervirulent clonal complexes, to adapt and tolerate slightly elevated concentrations of a commercial QAC (MIC 3 ppm; MBC 4 ppm) compared to the WT strains.

The six L. monocytogenes isolates evaluated were adaptable to a commercial QAC at 1 ppm above their WT MIC (2 ppm) and $\mathrm{MBC}$ (3 ppm). Previous studies using benzalkonium chloride (BC) compound noted a greater increase in MIC following adaptations. Similar to WT strains in the present study, Aase et al. (2000) reported five $L$. monocytogenes isolates with an initial MIC of 2 ppm; however, these isolates were then adapted to MICs of 6-7 ppm. It is of interest to note that other isolates tested within their study, with an initial MIC of 6 and 7 ppm, did not exceed 7 ppm MIC following the adaptation process (Aase et al., 2000). This suggests a potential plateau at which isolates are no longer readily adaptable, as we have observed in our study. Similar to Aase et al. (2000), a study by To et al. (2002) reported BC adaptation of L. monocytogenes isolates from 1 or $4 \mathrm{ppm} \mathrm{MIC} \mathrm{to} 6$ or $8 \mathrm{ppm}$ MIC, respectively. Others have reported adaptation to $\mathrm{BC}$ from $2 \mathrm{ppm}$ in WT strains 
TABLE 5 | Mutations in L. monocytogenes isolates following the adaptation to commercial quaternary ammonium compound-based sanitizer (cQAC; 3 ppm).

\begin{tabular}{|c|c|c|c|c|}
\hline \multirow[t]{2}{*}{ Isolate no. } & \multirow{2}{*}{$\begin{array}{c}\text { Total \# high } \\
\text { quality mutations }\end{array}$} & \multicolumn{3}{|c|}{ Transcriptional regulator fepR } \\
\hline & & Mutation & $\begin{array}{l}\text { AA } \\
\text { change }\end{array}$ & $\begin{array}{l}\text { Nucleotide } \\
\text { position }\end{array}$ \\
\hline WRLP354 & $\mathrm{n} / \mathrm{m}^{a}$ & & & \\
\hline WRLP380 & 2 & $G>A^{b}$ & Pro $>$ Ser & 322 \\
\hline WRLP394 & 4 & $\begin{array}{l}1 \text { nt deletion } \\
A>T \\
G>T\end{array}$ & Leu > PMSC & $\begin{array}{l}288 \\
291 \\
292\end{array}$ \\
\hline WRLP483 & 2 & $\begin{array}{l}C>A^{b} \\
G>T^{c}\end{array}$ & $\begin{array}{l}\text { Pro }>\text { Gln } \\
\text { Glu }>\text { PMSC }\end{array}$ & $\begin{array}{l}320 \\
526\end{array}$ \\
\hline WRLP530 & 3 & $33 \mathrm{nt}$ deletion & & 40 \\
\hline WRLP533 & 2 & $\begin{array}{l}C>T^{b} \\
6 \text { nt deletion }\end{array}$ & His $>$ Tyr & $\begin{array}{l}121 \\
126-131\end{array}$ \\
\hline
\end{tabular}

${ }^{a} n / m$, no mutation.

${ }^{b}$ Mutation resulted in a change in amino acid (AA) sequence.

${ }^{c}$ Mutation resulted in a premature stop codon (PMSC).

to 10 ppm MIC in adapted strains (Yu et al., 2018). While these adaptations achieved a higher MIC than was observed in the present study, additional components of the formulated commercial QAC product may have inhibited the adaptation from progressing to $\mathrm{BC}$-adaptation levels. In fact, the majority of published studies on QAC and Listeria spp. have been performed using $\mathrm{BC}$ as opposed to commercial sanitizer preparations, such as the cQAC used in this study. BC contains an alkyl chain length distribution from C8 to C18. Previous research using narrower alkyl chain lengths has determined that Grampositive bacteria, such as L. monocytogenes, are most affected at chain lengths of $\mathrm{C} 12-\mathrm{C} 14$ whereas longer chain lengths of C14-C16 are more effective against Gram-negative bacteria, such as Escherichia coli (Gilbert and Moore, 2005). Commercial QAC products have been formulated to contain QACs with a specific distribution of alkyl chain lengths (e.g., C14: 50\%, C12: $40 \%$, and C16: $10 \%$ ) to optimize antimicrobial activity. These may also be formulated with additional ingredients, including divalent chelators like EDTA, that assist in destabilizing the bacterial cell membrane structures (Gilbert and Moore, 2005). The distribution of alkyl chain lengths and other additives in the commercial product formulation likely influence adaptation and complicate comparing MIC and MBC values from independent studies.

The primary mechanism of QAC adaptation and tolerance is largely attributed to the presence and upregulation of specific efflux pumps (Aase et al., 2000; Romanova et al., 2006; Kovacevic et al., 2013; Yu et al., 2018). Previous studies have reported the assistance of QAC specific efflux systems playing a role in resistance or adaptation, specifically the $\operatorname{bcr} A B C$ cassette (Elhanafi et al., 2010; Dutta et al., 2013), emrE (Kovacevic et al., 2016), emrC (Kremer et al., 2017), qacC and qacH (Müller et al., 2013). cQAC tolerance of the adapted strains in the present study was lost with the addition of reserpine, indicating that efflux systems are playing a significant role in the experimentally achieved cQAC tolerance. While the previously reported genomic analyses of six WT strains examined here (Bland et al., 2021) revealed the lack of efflux pumps commonly linked to increased tolerance to QAC (e.g., bcrABC, qacC, $q a c H$, emrE, or emrC), the restoration of the WT phenotype in the presence of reserpine, a known efflux pump inhibitor, suggests the role of other efflux pumps in the cQAC adaptation in these isolates.

Research studies have reported that two efflux pumps, $m d r L$ and $l d e$, ubiquitous in L. monocytogenes (Romanova et al., 2006; Yu et al., 2018), are upregulated following the exposure or adaptation to QAC, suggesting their role in tolerance toward the compound. Yu et al. (2018) found that the relative expression of $m d r L$ was significantly higher in six L. monocytogenes strains following adaptation to a QAC. In contrast, Jiang et al. (2018) reported that MICs were not affected by the absence of lde in a deletion mutant. While it can be speculated that $m d r L$ and lde may have played a role in adaptation, as they were both present in our studied isolates, there are additional, more broadly found multidrug resistant (MDR) efflux systems in Gram-positive bacteria that may be assisting in tolerance and cross-resistance (Tamburro et al., 2015; Schindler and Kaatz, 2016; Meier et al., 2017; Du et al., 2018). Some of these major families of MDR efflux systems have been described by Schindler and Kaatz (2016) including: (1) ATP-binding cassette, (2) major facilitator superfamily (lde), (3) multidrug and toxic extrusion systems ( $m d r L)$, (4) small multidrug resistance, and (5) resistance nodulation cell division (RND) family.

Other mechanisms of QAC tolerance have been explored in isolates without known efflux pumps associated with QACs. In a study by Meier et al. (2017) 45 L. monocytogenes isolates were found to possess high tolerance to BC (MIC > $20 \mathrm{ppm}$ ). When exposed to reserpine, only four isolates exhibited reduced tolerance to BC. They suggested that the majority of their BCtolerant strains did not rely on efflux systems. Studies have suggested that the decrease in cell membrane permeability plays an important role in the reduction of tolerance to QACs (McDonnell and Russell, 1999; To et al., 2002; Wessels and Ingmer, 2013) and chlorine and selective antibiotics (Bansal et al., 2018). An increase in the size (cells were elongated and filamentous) and a shift in the fatty acid composition of the cell (shift from shorter to longer fatty acid following adaptation-resulting in decrease in fluidity of the cell), was reported following $\mathrm{BC}$ adaptation, suggesting changes in the cell structure/membrane as a possible tolerance mechanism (To et al., 2002). While outside the scope of the data presented here, due to the lack of major efflux systems that have previously been linked to assisting in QAC tolerance development, it would be of interest to consider changes in cellular permeability and fatty acid composition in our isolates as a possible mechanism aiding the adaptation and cross resistance process.

Following cQAC adaptation, we observed a shift in susceptibility to CIP, KAN, NOV, PEN, and to a lesser degree CLI and CHL amongst the six L. monocytogenes strains tested. In previous studies, L. monocytogenes adapted to QAC resulted in phenotypic changes comparable to those seen in the present 
study with some minor differences. Similar to our results, the shift in susceptibility toward CIP following QAC adaptation has been described by Rakic-Martinez et al. (2011) and Yu et al. (2018), who both reported an increase in MIC of CIP following adaptation to BC. The decreased susceptibility to KAN following BC adaptation reported by Romanova et al. (2006) for $3 / 4$ tested isolates is similar to what we observed here, with the shift from sensitive to intermediate AMR profiles in 3/6 isolates. In contrast, Yu et al. (2018) did not see any changes in susceptibility to KAN in $\mathrm{BC}$-adapted isolates. These data suggest that the adaptive mechanisms for KAN differ among isolates.

Quaternary ammonium compound and CIP tolerance/resistance has been previously reported (RakicMartinez et al., 2011; Kovacevic et al., 2013), and appears to be bidirectional, where isolates adapted to QAC become more resistant to CIP and isolates adapted to CIP become more tolerant to QAC. CIP is a fluoroquinolone; its mechanism of action involves preventing DNA separation prior to cell division. Resistance to quinolones in Gram-positive bacteria is typically due to mutations in the intercellular targets of the compound, gyrA and gyrB (DNA gyrase), parC and parE (topoisomerase IV) or decreased uptake into the cell via efflux pumps (Lampidis et al., 2002; Jiang et al., 2018). Jiang et al. (2018) found no mutations in the genes targeted by quinolones. However, transcription levels of $l d e$ increased across the four CIP adapted mutants, suggesting that the resistance development was largely due to decreased uptake into the cell (Jiang et al., 2018). Others have suggested that point mutations in fepR (regulator for fepA) are in part responsible for resistance to CIP (Wilson et al., 2018). FepA, a part of the multidrug and toxic compound extrusion (MATE) family of efflux pumps, is regulated by fepR (Guerin et al., 2014). When MICs of various antibiotics of WT L. monocytogenes and its fepR deletion mutant were compared, Guerin et al. (2014) reported susceptibility changes only in fluroquinolones. They also saw a change in BC susceptibility in the fepR deletion mutant, with an MIC increase from 4 to $8 \mathrm{ppm}$, suggesting that overexpression of the fepA efflux pump may play a role in BC tolerance. An Australian study by Wilson et al. (2018) found 2/100 L. monocytogenes possessing CIP resistance; in one of the isolates CIP resistance was linked to a mutation at nucleotide position 181 in $f e p R$, resulting in PMSC. A point mutation in $f e p R$ was also seen in the other CIP resistant isolate, at nucleotide position 170, leading to an amino acid change (alanine to glycine). However, this mutation did not result in a PMSC, but rather this isolate possessed a full length $f e p R$ (Wilson et al., 2018). Similarly, our results found point mutations at different locations in $f e p R$, some of which resulted in a PMSC and others that did not. In our isolates, both the location of the mutation as well as the resulting PMSC were downstream of what was previously seen by Guerin et al. (2014) and Wilson et al. (2018), resulting in a slightly longer gene. The multiple and independent mutations in $f e p R$ in the current study are strong evidence that loss of fepR function is important for L. monocytogenes adapting to CQAC. However, definite proof of the role of $f e p R$ and the level of its contribution to QAC adaptation would require deletion and complementation mutants.
Previous studies evaluating cross-resistance of QAC and antibiotics amongst $L$. monocytogenes have not, to our knowledge, evaluated NOV. NOV is less commonly used due to its decreased efficacy, but it has synergistic activity with tetracyclines, and can be used as an alternative to penicillin (May et al., 2017). Our results suggest that there is a relationship between adaptive QAC tolerance and NOV resistance, as a shift in susceptibility was seen across all six isolates. In particular, a dramatic shift from susceptible to resistant classification was observed in two strains, WRLP354 and WRLP530. Similar to quinolones, NOV inhibits gyrB subunit of the bacterial DNA gyrase enzyme involved in energy transduction (Hof et al., 1997; May et al., 2017). The phenotypic cross-resistance can be associated with mutations in the target for NOV or changes in cellular structure to reduce accumulation within the cell. In Gram-positive bacteria, such as Bacillus lincheniformis, morphological changes have been associated with NOV resistance. Specifically, in the presence of NOV the cells grow as long filaments as opposed to long chains (Robson and Baddiley, 1977). In Gram-negative bacteria, NOV resistance has been associated with a two-component regulatory system (baeSR), activating an efflux pump system ( $m d t A B C)$. This is believed to lead to reduced NOV accumulation within the cell (Baranova and Nikaido, 2002; Nagakubo et al., 2002). In our isolates, we did not observe mutations in $\operatorname{gyr} A B$. While morphological changes were not investigated, they cannot be ruled out as potential mechanisms aiding the resistance (Robson and Baddiley, 1977).

Our results also indicate that $c Q A C$ adaptation affects PEN resistance, with decreased zones of inhibition observed. PEN is a B-lactam antibiotic, similar to AMP. However, unlike AMP, which is frequently used in the treatment of listeriosis, PEN is not typically used due to increasing reports of microbial resistance (Wilson et al., 2018). The isolates tested in this study were all classified as either having intermediate or resistant PEN profiles. However, while we observed a slight decrease in the zone of inhibition for AMP following $\mathrm{CQAC}$ adaptation, all tested isolates remained well within the susceptible classification (Figure 2). B-lactam antibiotics, such as PEN, work to inhibit cell wall biosynthesis through interactions with penicillinbinding proteins (PBP). Listeria spp. have five $\mathrm{PBP}$, and $\mathrm{PBP} 3$ is largely the target of B-lactams (Vicente et al., 1990; Hof et al., 1997). Insertional mutagenesis in associated genes in L. monocytogenes EGDe (e.g., lmo0441, lmo0504, lmo1438, and Imo2229) resulted in acquired resistance toward PEN (Guinane et al., 2006). In S. pneumoniae, PEN resistance typically occurs from recombination events leading to mosaic PBP, which in turn can lead to reduced affinity for PEN and diminished effect on the cell (Blair et al., 2015).

It has been reported that exposure to QAC can increase the expression of virulence genes, such as prfA and inlA (Kastbjerg et al., 2010). Since cQAC adaptation impacted AMR profiles and led to genomic changes in some of our studied isolates, it was prudent to explore if virulence genes were affected, especially since our isolates belong to hypervirulent clonal complexes (Maury et al., 2016). While we did not look at the expression of virulence genes following $\mathrm{CQAC}$ adaptation, it is of note that 
no mutations in L. monocytogenes virulence genes were observed following cQAC adaptation.

\section{CONCLUSION}

Collectively, our data demonstrate the potential for L. monocytogenes isolates to develop cross-resistance between a cQAC and antibiotics representing different classes. While the increased MIC and MBC of CQAC-adapted isolates remained well below the manufacturer recommended concentration for the commercial product, it is not uncommon for bacterial cells to be exposed to lower or sublethal concentrations of sanitizers in the processing environment either by dilution, presence of organic matter (e.g., decreasing the efficacy of the sanitizer), or issues with the hygienic design of equipment or facility (e.g., resulting in microbial niches, biofilm formation or dilution effect of sanitizer). The isolates in the present study were adaptable to $1 \mathrm{ppm}$ higher than the WT MIC. This minimal MIC increase suggests that adaptation of L. monocytogenes to a degree that would render the cQAC product ineffective is not likely in commercial settings. However, the present study was done with planktonic cells and so the additional tolerance and protection provided in biofilms, both in terms of initial susceptibility and degree of adaptation, likely differs. In a processing facility, the circumstances that may allow for exposure to minimal concentrations of CQAC are often associated with improper cleaning and inadequate sanitation practices that may not be addressing microbial niches. This can lower the effectiveness of the sanitizer or may be resulting in a dilution effect in some areas of the facility. Our data highlight that sublethal exposures to cQACs could have deleterious effects if adapted L. monocytogenes strains become implicated in human illness that requires antibiotic treatment. The potential for L. monocytogenes to develop cross-resistance to clinically relevant antibiotics following minimal adaptation to a formulated CQAC product is especially concerning. In particular, this trend amongst genotypes that are classified as hypervirulent and frequently involved in cases of listeriosis highlights the need to better understand the effect that sanitizer exposures and low-level adaptations may have on the AMR development and a potential public health risk.

\section{REFERENCES}

Aase, B., Sundheim, G., Langsrud, S., and Rørvik, L. M. (2000). Occurrence of and a possible mechanism for resistance to a quaternary ammonium compound in Listeria monocytogenes. Int. J. Food Microbiol. 62, 57-63. doi: 10.1016/s01681605(00)00357-3

Amsalu, A., Sapula, S. A., De Barros Lopes, M., Hart, B. J., Nguyen, A. H., Drigo, B., et al. (2020). Efflux pump-driven antibiotic and biocide cross-resistance in Pseudomonas aeruginosa isolated from different ecological niches: a case study in the development of multidrug resistance in environmental hotspots. Microorganisms 8:1647. doi: 10.3390/microorganisms8111647

Bansal, M., Nannapaneni, R., Sharma, C. S., and Kiess, A. (2018). Listeria monocytogenes response to sublethal chlorine induced oxidative stress on homologous and heterologous stress adaptation. Front Microbiol. 9:2050. doi: $10.3389 /$ fmicb. 2018.02050

Baranova, N., and Nikaido, H. (2002). The baeSR two-component regulatory system activates transcription of the yegMNOB (mdtABCD) transporter gene

\section{DATA AVAILABILITY STATEMENT}

The dataset presented in this study can be found in the NCBI Sequence Read Archive (SRA) under BioProject PRJNA771688.

\section{AUTHOR CONTRIBUTIONS}

JW-C and JK: conceptualization. RB and ER: data curation. $\mathrm{RB}, \mathrm{AW}, \mathrm{JW}-\mathrm{C}$, and JK: formal analysis and methodology. $\mathrm{JK}$ : funding acquisition and project administration. AW, JC, JW-C, and JK: supervision and writing - review and editing. AW: validation. RB: visualization and writing - original draft. All authors have read and agreed to the published version of the manuscript.

\section{FUNDING}

This research was funded by the OSU Agricultural Research Foundation grant (ARF\#9148A).

\section{ACKNOWLEDGMENTS}

We also acknowledge Grant and Alice Schoenhard Faculty Excellence fund awarded to $\mathrm{JK}$ for partial graduate assistantship support for RB. We extend our thanks to the Center for Quantitative Life Sciences, Oregon State University for WGS services. We also thank the Department of Botany and Plant Pathology for its support of the computational infrastructure.

\section{SUPPLEMENTARY MATERIAL}

The Supplementary Material for this article can be found online at: https://www.frontiersin.org/articles/10.3389/fmicb. 2021.782920/full\#supplementary-material

cluster in Escherichia coli and increases its resistance to novobiocin and deoxycholate. J. Bacteriol. 184, 4168-4176. doi: 10.1128/JB.184.15.4168-4176. 2002

Baranyi, J., and Roberts, T. A. (1994). A dynamic approach to predicting bacterial growth in food. Int. J. Food Microbiol. 23, 277-294.

Blair, J. M., Webber, M. A., Baylay, A. J., Ogbolu, D. O., and Piddock, L. J. (2015). Molecular mechanisms of antibiotic resistance. Nat. Rev. Microbiol. 13, 42-51. doi: $10.1038 /$ nrmicro3380

Bland, R., Johnson, J., Waite-Cusic, J., Weisberg, A. J., Riutta, E. R., Chang, J. H., et al. (2021). Application of whole genome sequencing to understand diversity and presence of genes associated with sanitizer tolerance in Listeria monocytogenes from produce handling sources. Foods 10:2454. doi: 10.3390/ foods 10102454

Boucher, C., Waite-Cusic, J., Stone, D., and Kovacevic, J. (2021). Relative performance of commercial citric acid and quaternary ammonium sanitizers against Listeria monocytogenes under conditions relevant to food industry. Food Microbiol. 97:103752. doi: 10.1016/j.fm.2021.103752 
Braoudaki, M., and Hilton, A. C. (2004). Adaptive resistance to biocides in Salmonella enterica and Escherichia coli $\mathrm{O} 157$ and cross-resistance to antimicrobial agents. J. Clin. Microbiol. 42, 73-78. doi: 10.1128/JCM.42.1.7378.2004

Buchanan, R. L., Gorris, L. G., Hayman, M. M., Jackson, T. C., and Whiting, R. C. (2017). A review of Listeria monocytogenes: an update on outbreaks, virulence, dose-response, ecology, and risk assessments. Food Control 75, 1-13. doi: 10.1016/j.foodcont.2016.12.016

Cherifi, T., Carrillo, C., Lambert, D., Miniai, I., Quessy, S., Lariviere-Gauthier, G., et al. (2018). Genomic characterization of Listeria monocytogenes isolates reveals that their persistence in a pig slaughterhouse is linked to the presence of benzalkonium chloride resistance genes. BMC Microbiol. 18:220. doi: 10.1186/ s12866-018-1363-1369

Cingolani, P., Platts, A., Wang le, L., Coon, M., Nguyen, T., Wang, L., et al. (2012). A program for annotating and predicting the effects of single nucleotide polymorphisms, SnpEff: SNPs in the genome of Drosophila melanogaster strain w1118; iso-2; iso-3. Fly (Austin) 6, 80-92. doi: 10.4161/fly.19695

CLSI (2015). Methods for Antimicrobial Dilution and Disk Suseptibility Testing of Infrequently Isolated or Fastidious Bacteria. CLSI guidline M45. 3rd Edn Wayne, PA: Clinical and Laboratory Standards Institute.

Cruz, C. D., and Fletcher, G. C. (2012). Assessing manufacturers' recommended concentrations of commercial sanitizers on inactivation of Listeria monocytogenes. Food Control 26, 194-199. doi: 10.1016/j.foodcont.2012. 01.041

de Noordhout, C. M., Devleesschauwer, B., Angulo, F. J., Verbeke, G., Haagsma, J., Kirk, M., et al. (2014). The global burden of listeriosis: a systematic review and meta-analysis. Lancet Infect. Dis. 14, 1073-1082. doi: 10.1016/S1473-3099(14) 70870-9

Du, D., Wang-Kan, X., Neuberger, A., van Veen, H. W., Pos, K. M., Piddock, L. J., et al. (2018). Multidrug efflux pumps: structure, function and regulation. Nat. Rev. Microbiol. 16, 523-539. doi: 10.1038/s41579-018-0048-46

Dutta, V., Elhanafi, D., and Kathariou, S. (2013). Conservation and distribution of the benzalkonium chloride resistance cassette bcrABC in Listeria monocytogenes. Appl. Environ. Microbiol. 79, 6067-6074. doi: 10.1128/AEM. $01751-1713$

Edgar, R. C. (2004). MUSCLE: a multiple sequence alignment method with reduced time and space complexity. BMC Bioinformatics 5:113. doi: 10.1186/1471-21055- 113

Eggertsson, H. P., Jonsson, H., Kristmundsdottir, S., Hjartarson, E., Kehr, B., Masson, G., et al. (2017). Graphtyper enables population-scale genotyping using pangenome graphs. Nat. Genet. 49, 1654-1660. doi: 10.1038/ng.3964

Elhanafi, D., Dutta, V., and Kathariou, S. (2010). Genetic characterization of plasmid-associated benzalkonium chloride resistance determinants in a Listeria monocytogenes strain from the 1998-1999 outbreak. Appl. Environ. Microbiol. 76, 8231-8238. doi: 10.1128/AEM.02056-2010

Farber, J. M., Zwietering, M., Wiedmann, M., Schaffner, D., Hedberg, C. W., Harrison, M. A., et al. (2020). Alternative approaches to the risk management of Listeria monocytogenes in low risk foods. Food Control 123:107601. doi: 10.1016/j.foodcont.2020.107601

Freitag, N. E., Port, G. C., and Miner, M. D. (2009). Listeria monocytogenes from saprophyte to intracellular pathogen. Nat. Rev. Microbiol. 7, 623-628. doi: $10.1038 /$ nrmicro2171

Gallagher, D. L., Ebel, E. D., and Kause, J. R. (2003). FSIS Risk Assessment for Listeria monocytogenes in Deli Meats. Maryland, MD: Food Safety and Inspection Service, USDA.

Gilbert, P., and Moore, L. (2005). Cationic antiseptics: diversity of action under a common epithet. J. Appl. Microbiol. 99, 703-715. doi: 10.1111/j.1365-2672. 2005.02664.x

Gnanadhas, D. P., Marathe, S. A., and Chakravortty, D. (2013). Biocides-resistance, cross-resistance mechanisms and assessment. Expert Opin. Investig. Drugs 22, 191-206. doi: 10.1517/13543784.2013.748035

Godreuil, S., Galimand, M., Gerbaud, G., Jacquet, C., and Courvalin, P. (2003). Efflux pump lde is associated with fluoroquinolone resistance in Listeria monocytogenes. Antimicrob. Agents Chemother. 47, 704-708. doi: 10.1128/AAC. 47.2.704-708.2003

Gray, M. J., Freitag, N. E., and Boor, K. J. (2006). How the bacterial pathogen Listeria monocytogenes mediates the switch from environmental Dr. Jekyll to pathogenic Mr. Hyde. Infect. Immun. 74, 2505-2512. doi: 10.1128/IAI.74.5. 2505-2512.2006

Guerin, F., Galimand, M., Tuambilangana, F., Courvalin, P., and Cattoir, V. (2014). Overexpression of the novel MATE fluoroquinolone efflux pump FepA in Listeria monocytogenes is driven by inactivation of its local repressor FepR. PLoS One 9:e106340. doi: 10.1371/journal.pone.0106340

Guinane, C. M., Cotter, P. D., Ross, R. P., and Hill, C. (2006). Contribution of penicillin-binding protein homologs to antibiotic resistance, cell morphology, and virulence of Listeria monocytogenes EGDe. Antimicrob. Agents Chemother. 50, 2824-2828. doi: 10.1128/AAC.00167-166

Hamon, M., Bierne, H., and Cossart, P. (2006). Listeria monocytogenes: a multifaceted model. Nat. Rev. Microbiol. 4, 423-434. doi: 10.1038/nrmicro1413

Heir, E., Sundheim, G., and Holck, A. L. (1999). The qacG gene on plasmid pST94 confers resistance to quaternary ammonium compounds in staphylococci isolated from the food industry. J. Appl. Microbiol. 86, 378-388. doi: 10.1046/ j.1365-2672.1999.00672.x

Hof, H. (2004). An update on the medical management of listeriosis. Expert Opin. Pharmacother. 5, 1727-1735. doi: 10.1517/14656566.5.8.1727

Hof, H., Nichterlein, T., and Kretschmar, M. (1997). Management of listeriosis. Clin. Microbiol. Rev. 10, 345-357. doi: 10.1128/CMR.10.2.345

Hurley, D., Luque-Sastre, L., Parker, C. T., Huynh, S., Eshwar, A. K., Nguyen, S. V., et al. (2019). Whole-genome sequencing-based characterization of 100 Listeria monocytogenes isolates collected from food processing environments over a four-year period. $m$ Sphere 4:e00252-19. doi: 10.1128/mSphere.00252-219

Jett, B. D., Hatter, K. L., Huycke, M. M., and Gilmore, M. S. (1997). Simplified agar plate method for quantifying viable bacteria. BioTechniques 23, 648-650. doi: 10.2144/97234bm22

Jiang, X., Yu, T., Xu, P., Xu, X., Ji, S., Gao, W., et al. (2018). Role of efflux pumps in the in vitro development of ciprofloxacin resistance in Listeria monocytogenes. Front. Microbiol. 9:2350. doi: 10.3389/fmicb.2018.02350

Jorgensen, J., Bland, R., Waite-Cusic, J., and Kovacevic, J. (2021). Diversity and antimicrobial resistance of Listeria spp. and L. monocytogenes clones from produce handling and processing facilities in the Pacific Northwest. Food Control 123:107665. doi: 10.1016/j.foodcont.2020.107665

Jorgensen, J., Waite-Cusic, J., and Kovacevic, J. (2020). Prevalence of Listeria spp. in produce handling and processing facilities in the Pacific Northwest. Food Microbiol. 90:103468. doi: 10.1016/j.fm.2020.103468

Kastbjerg, V. G., Larsen, M. H., Gram, L., and Ingmer, H. (2010). Influence of sublethal concentrations of common disinfectants on expression of virulence genes in Listeria monocytogenes. Appl. Environ. Microbiol. 76, 303-309. doi: 10.1128/AEM.00925-929

Kim, S. W., Haendiges, J., Keller, E. N., Myers, R., Kim, A., Lombard, J. E., et al. (2018). Genetic diversity and virulence profiles of Listeria monocytogenes recovered from bulk tank milk, milk filters, and milking equipment from dairies in the United States (2002 to 2014). PLoS One 13:e0197053. doi: 10.1371/ journal.pone.0197053

Kode, D., Nannapaneni, R., Bansal, M., Chang, S., Cheng, W. H., Sharma, C. S., et al. (2021). Low-level tolerance to fluoroquinolone antibiotic ciprofloxacin in QAC-adapted subpopulations of Listeria monocytogenes. Microorganisms 9. doi: 10.3390/microorganisms 9051052

Kovacevic, J., Sagert, J., Wozniak, A., Gilmour, M. W., and Allen, K. J. (2013). Antimicrobial resistance and co-selection phenomenon in Listeria spp. recovered from food and food production environments. Food Microbiol. 34, 319-327. doi: 10.1016/j.fm.2013.01.002

Kovacevic, J., Ziegler, J., Walecka-Zacharska, E., Reimer, A., Kitts, D. D., and Gilmour, M. W. (2016). Tolerance of Listeria monocytogenes to quaternary ammonium sanitizers is mediated by a novel efflux pump encoded by emrE. Appl. Environ. Microbiol. 82, 939-953. doi: 10.1128/AEM.03741-3715

Kremer, P. H., Lees, J. A., Koopmans, M. M., Ferwerda, B., Arends, A. W., Feller, M. M., et al. (2017). Benzalkonium tolerance genes and outcome in Listeria monocytogenes meningitis. Clin. Microbiol. Infect. 23, 265.e1-265.e7. doi: 10. 1016/j.cmi.2016.12.008

Lampidis, R., Kostrewa, D., and Hof, H. (2002). Molecular characterization of the genes encoding DNA gyrase and topoisomerase IV of Listeria monocytogenes J. Antimicrob. Chemother. 49, 917-924. doi: 10.1093/jac/dkf065

Langsrud, S., Sundheim, G., and Holck, A. L. (2004). Cross-resistance to antibiotics of Escherichia coli adapted to benzalkonium chloride or exposed to 
stress-inducers. J. Appl. Microbiol. 96, 201-208. doi: 10.1046/j.1365-2672.2003.0 2140.x

Liao, J., Guo, X., Weller, D. L., Pollak, S., Buckley, D. H., Wiedmann, M., et al. (2021). Nationwide genomic atlas of soil-dwelling Listeria reveals effects of selection and population ecology on pangenome evolution. Nat. Microbiol. 6, 1021-1030. doi: 10.1038/s41564-021-00935-937

Linke, K., Ruckerl, I., Brugger, K., Karpiskova, R., Walland, J., Muri-Klinger, S., et al. (2014). Reservoirs of Listeria species in three environmental ecosystems. Appl. Environ. Microbiol. 80, 5583-5592. doi: 10.1128/AEM.01018-1014

Martínez-Suárez, J. V., Ortiz, S., and López-Alonso, V. (2016). Potential impact of the resistance to quaternary ammonium disinfectants on the persistence of Listeria monocytogenes in food processing environments. Front. Microbiol. 7:638. doi: 10.3389/fmicb.2016.00638

Maury, M. M., Tsai, Y.-H., Charlier, C., Touchon, M., Chenal-Francisque, V., Leclercq, A., et al. (2016). Uncovering Listeria monocytogenes hypervirulence by harnessing its biodiversity. Nat. Genet. 48:308. doi: 10.1038/ng.3501

May, J. M., Owens, T. W., Mandler, M. D., Simpson, B. W., Lazarus, M. B., Sherman, D. J., et al. (2017). The antibiotic novobiocin binds and activates the ATPase that powers lipopolysaccharide transport. J. Am. Chem. Soc. 139, 17221-17224. doi: 10.1021/jacs.7b07736

McDonnell, G., and Russell, A. D. (1999). Antiseptics and disinfectants: activity, action, and resistance. Clin. Microbiol. Rev. 12, 147-179. doi: 10.1128/CMR.12. 1.147

Meier, A. B., Guldimann, C., Markkula, A., Pöntinen, A., Korkeala, H., and Tasara, T. (2017). Comparative phenotypic and genotypic analysis of Swiss and Finnish Listeria monocytogenes isolates with respect to benzalkonium chloride resistance. Front. Microbiol. 8:397. doi: 10.3389/fmicb.2017.00397

Møretrø, T., Schirmer, B. C., Heir, E., Fagerlund, A., Hjemli, P., and Langsrud, S. (2017). Tolerance to quaternary ammonium compound disinfectants may enhance growth of Listeria monocytogenes in the food industry. Int. J. Food Microbiol. 241, 215-224. doi: 10.1016/j.ijfoodmicro.2016.10.025

Müller, A., Rychli, K., Muhterem-Uyar, M., Zaiser, A., Stessl, B., Guinane, C. M., et al. (2013). Tn6188-a novel transposon in Listeria monocytogenes responsible for tolerance to benzalkonium chloride. PLoS One 8:e76835. doi: 10.1371/ journal.pone.0076835

Nagakubo, S., Nishino, K., Hirata, T., and Yamaguchi, A. (2002). The putative response regulator BaeR stimulates multidrug resistance of Escherichia coli via a novel multidrug exporter system. MdtABC. J. Bacteriol. 184, 4161-4167. doi: 10.1128/JB.184.15.4161-4167.2002

Office of Chemical Safety and Pollution Prevention [OCSPP] (2012). Product Performance Test Guidelines: OCSPP 810.2300 Sanitizers for Use on Hard Surfaces - Efficacy Data Recommendations [EPA 712-C-07-091]. Washington, D.C: Office of Chemical Safety and Pollution Prevention

Prazak, A. M., Murano, E. A., Mercado, I., and Acuff, G. R. (2002). Antimicrobial resistance of Listeria monocytogenes isolated from various cabbage farms and packing sheds in Texas. J. Food Prot. 65, 1796-1799. doi: 10.4315/0362-028X65.11.1796

Rakic-Martinez, M., Drevets, D. A., Dutta, V., Katic, V., and Kathariou, S. (2011). Listeria monocytogenes strains selected on ciprofloxacin or the disinfectant benzalkonium chloride exhibit reduced susceptibility to ciprofloxacin, gentamicin, benzalkonium chloride, and other toxic compounds. Appl. Environ. Microbiol. 77, 8714-8721. doi: 10.1128/AEM.059415911

Robson, R. L., and Baddiley, J. (1977). Morphological changes associated with novobiocin resistance in Bacillus licheniformis. J. Bacteriol. 129, 1045-1050. doi: 10.1128/jb.129.2.1045-1050.1977

Roedel, A., Dieckmann, R., Brendebach, H., Hammerl, J., Kleta, S., Noll, M., et al. (2019). Biocide-tolerant Listeria monocytogenes isolates from German food production plants do not show cross-resistance to clinically relevant antibiotics. Appl. Environ. Microbiol. 85:e01253-19. doi: 10.1128/AEM.012531219

Romanova, N., Wolffs, P., Brovko, L., and Griffiths, M. (2006). Role of efflux pumps in adaptation and resistance of Listeria monocytogenes to benzalkonium chloride. Appl. Environ. Microbiol. 72, 3498-3503. doi: 10.1128/AEM.72.5. 3498-3503.2006

Scallan, E., Hoekstra, R. M., Angulo, F. J., Tauxe, R. V., Widdowson, M. A., Roy, S. L., et al. (2011). Foodborne illness acquired in the United States-major pathogens. Emerg. Infect. Dis. 17, 7-15. doi: 10.3201/eid1701.P11101

SCENIHR (2009). Assessment of the Antibiotic Resistance Effects on Biocides. Brussels: Scientific Committees. European Commission.

Schindler, B. D., and Kaatz, G. W. (2016). Multidrug efflux pumps of Gram-positive bacteria. Drug Resist. Updates 27, 1-13. doi: 10.1016/j.drup.2016.04.003

Sullivan, G., and Wiedmann, M. (2020). Detection and prevalence of Listeria in US produce packinghouses and fresh-cut facilities. J. Food Prot. 83, 1656-1666. doi: 10.4315/JFP-20-094

Tamburro, M., Ripabelli, G., Vitullo, M., Dallman, T. J., Pontello, M., Amar, C. F., et al. (2015). Gene expression in Listeria monocytogenes exposed to sublethal concentration of benzalkonium chloride. Comp. Immunol. Microbiol. Infect. Dis. 40, 31-39. doi: 10.1016/j.cimid.2015.03.004

To, M. S., Favrin, S., Romanova, N., and Griffiths, M. W. (2002). Postadaptational resistance to benzalkonium chloride and subsequent physicochemical modifications of Listeria monocytogenes. Appl. Environ. Microbiol. 68, 5258-5264. doi: 10.1128/AEM.68.11.5258-5264.2002

Vicente, M. F., Perez-Daz, J. C., Baquero, F., Angel de Pedro, M., and Berenguer, J. (1990). Penicillin-binding protein 3 of Listeria monocytogenes as the primary lethal target for beta-lactams. Antimicrob. Agents Chemother. 34, 539-542. doi: 10.1128/AAC.34.4.539

Wessels, S., and Ingmer, H. (2013). Modes of action of three disinfectant active substances: a review. Regul. Toxicol. Pharmacol. 67, 456-467. doi: 10.1016/j. yrtph.2013.09.006

Wilson, A., Gray, J., Chandry, P. S., and Fox, E. M. (2018). Phenotypic and genotypic analysis of antimicrobial resistance among Listeria monocytogenes isolated from Australian food production chains. Genes (Basel) 9:80. doi: 10. 3390/genes 9020080

Yu, T., Jiang, X., Zhang, Y., Ji, S., Gao, W., and Shi, L. (2018). Effect of benzalkonium chloride adaptation on sensitivity to antimicrobial agents and tolerance to environmental stresses in Listeria monocytogenes. Front. Microbiol. 9:2906. doi: 10.3389/fmicb.2018.02906

Conflict of Interest: The authors declare that the research was conducted in the absence of any commercial or financial relationships that could be construed as a potential conflict of interest.

Publisher's Note: All claims expressed in this article are solely those of the authors and do not necessarily represent those of their affiliated organizations, or those of the publisher, the editors and the reviewers. Any product that may be evaluated in this article, or claim that may be made by its manufacturer, is not guaranteed or endorsed by the publisher.

Copyright (c) 2022 Bland, Waite-Cusic, Weisberg, Riutta, Chang and Kovacevic. This is an open-access article distributed under the terms of the Creative Commons Attribution License (CC BY). The use, distribution or reproduction in other forums is permitted, provided the original author(s) and the copyright owner(s) are credited and that the original publication in this journal is cited, in accordance with accepted academic practice. No use, distribution or reproduction is permitted which does not comply with these terms. 